\title{
Review of Monitoring Methods for Submicronsized Particulates Emission in Coal-fired Power Plants
}

\author{
Chen Yiwei, Li Gengda, Sun Zhenxin, Guo Hua, Liu Hanqiang, \\ Chen Haoran, Du Shuming, Yang Yang \\ Guodian New Energy Technology Research Institute, \\ Beijing Key Laboratory of Power Generation System Functional Material, \\ Changping District, Beijing 102209, China
}

\begin{abstract}
Particulates emission from coal-fired power plant is considered to be the main source of air pollutants. With the implementation of the new China national ambient air quality standard, monitoring and controlling particulate emission from coal-fired power plants are very important, and how to accurately measure the emission of particulate matters is the key point. There are two major challenges for monitoring methods: the determination of PM 10 and PM 2.5 emissions from coal-fired power plants and the measurement of the low concentration particulates in high humidity flue gas environment. Views of the above problems, all of the mainstream technologies for these two difficulties are reviewed in this paper, and both advantages and disadvantages of these methods are discussed as well. The result shows that: in high humidity flue gas, the relative accurate results of particulates concentration can be obtained by membrane filter method. Additionally, the direct sampling method of fine particulate matter based on source environment was widely used in daily monitoring and it can basically meet the needs of classification detection of fine particles in coal-fired power plant.
\end{abstract}

Key words: low concentration; test; high humidity; PM2.5; particulates

\section{INTRODUCTION}

The new China national ambient air quality standard has been published in 2012 and will be implemented in 2016. To meet the requirements in this new standard, monitoring and controlling PM 2.5 emission from coal-fired power plants are very important.

However, so far there is no national standard method on sampling PM 2.5 from coal-fired power plants. Meanwhile, with the implementation of Emission standard of air pollutants for thermal power plants (GB-13223-2011) and the use of new dust removal technology in china, effluent concentration of particulates arrives at under $10 \mathrm{mg} / \mathrm{Nm} 3$, even 1 $\mathrm{mg} / \mathrm{Nm} 3$, which is very difficult to detect, especially in high humidity environment of the power plant.
Therefore, how to accurately measure the content of the low concentration of particulates in high humidity environment has been an urgent technical problem. According to the above problems, different sampling and testing methods for PM 2.5 as well as particulates concentration test methods form coal-fired power plants relevant international standards were reviewed in this study. It includes the methods for PM 2.5 sampling in flue gas and the methods for PM 2.5 sampling after dilution. Both advantages and disadvantages of these methods were discussed and recommendations were given.

\section{MEASUREMENT OF LOW CONCENTRATION}

PARTICULATES CONTENT IN HIGH HUMIDITY FLUE GAS

Currently, the methods of monitoring particulates concentration can be classified into two classes: one is direct method, such as weighing method, direct method is the most basic and simplest method, and it needs no calibration so that it can get the absolute particulates concentration directly. The second method is indirect measurement method, that is to transfer the particulates concentration into electric signal according to the physicochemical property of dust particle, then make the particulates concentration in positive correlation relationship with electric signal in certain range, thus obtaining relative particulates concentration, then obtain the absolute particulates concentration by calibrating with weighing method.

\section{A. Direct measurement method:}

Direct measurement method (Or weighing method) is basic method to measure the particle mass concentration, it is simple, sampling with stipulated flow, and gather the particle floated in flue gas on high-performance filter membrane or filter cartridge, the mass difference before and after sampling represents the dust particle mass[1]. Traditional flue dust sampling method mainly adopts the way of filter 
cartridge sampling balance weighing (Refer to Methods of Performance Tests for Electrostatic Precipitators (GB /T 13931-2002)) [2], equal-area disk method is used at pipe to select proper sampling point for constant speed sampling, then record the sampling volume, temperature and humidity, pressure, and conduct drying and weighting of filter cartridge. There are many shortcomings when carrying out low concentration of dust emission with traditional flue dust sampling method, for example, long sampling time is needed, filter cartridge will be worn with weight loss, sampling nozzle is residual and so on. Therefore, to ensure the accuracy of measuring data, Laoying, Fujian Longking Environment Protection, Beijing Huayun Ante Technology Co., Ltd. have made a series of improvement on the traditional sampling method, and push out the membrane weighing method for low concentration of dust emission test.

The main improvement of membrane weighing method is the use of integrated low concentration sampling head, as shown in Fig. 1.1, the improved low concentration sampling spearhead adopts integrated sampling head and sampling nozzle, after sampling, the sampling nozzle and filter membrane are dried and weighted together, so as to avoid adhesion loss, also reduce the air leakage between sampling head and sampling nozzle, and reduce test error. To avoid the fine fiber falling on filter cartridge surface due to easy collision during carrying and transportation of sample, this will influence test result. The instrument manufacturer adopts filter membrane to replace filter cartridge, so as to obviously improve the sample parallelism and test accuracy, and the test shows that with high quality filter membrane, the measurement accuracy of such technology will be significantly improved ${ }^{[3]}$.

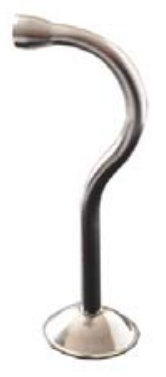

Fig.1.1 Sampling-head for low concentration of particulates

Other improvement measure includes larger flow of sampling pump to increase the sampling volume, increase the heat tracing system and strengthen gas tightness, etc. The measurement accuracy and parallelism of membrane weighing method after improvement is greatly strengthened (See table 1.1) [4]. Through measurement, the standard deviation of such method is within 0.13 .
TABLE 1.1 COMPARISON OF INFLUENCE FACTOR BEFORE AND

AFTER IMPROVEMENT OF LOW CONCENTRATION EMISSION TEST

\begin{tabular}{|c|c|c|c|}
\hline Items & $\begin{array}{l}\text { Cartridge } \\
\text { sampling }\end{array}$ & $\begin{array}{c}\text { Membran } \\
\text { e } \\
\text { sampling }\end{array}$ & $\begin{array}{c}\text { Improvement } \\
\text { measures }\end{array}$ \\
\hline $\begin{array}{l}\text { Adhesion loss } \\
\text { /mg }\end{array}$ & $0.5 \sim 2$ & 0 & Integrate design \\
\hline $\begin{array}{c}\text { Air } \\
\text { leakage } / \mathrm{L} \cdot \min \\
-1\end{array}$ & $0 \sim 5$ & 0 & $\begin{array}{c}\text { Optimize sampling } \\
\text { head }\end{array}$ \\
\hline $\begin{array}{l}\text { Transportation } \\
\text { losses /mg }\end{array}$ & $0 \sim 3$ & $0 \sim 0.3$ & $\begin{array}{l}\text { Use high-quality } \\
\text { filter membrane }\end{array}$ \\
\hline $\begin{array}{c}\text { Sampling } \\
\text { flow/ L·min-1 }\end{array}$ & $20 \sim 35$ & $\geq 60$ & Use large flow pump \\
\hline $\begin{array}{l}\text { Sampling } \\
\text { time/min }\end{array}$ & $86 \sim 150$ & $\leq 50$ & $\begin{array}{c}\text { Shorten sampling } \\
\text { time }\end{array}$ \\
\hline
\end{tabular}

\section{B. Indirect measurement method:}

The indirect measurement method can realize online continuously detecting, as a result, all the instruments adopted by power plant use the indirect measurement method. The main technical route can be divided into three types: 1) Direct extraction of high temperature gasification and the forward scattering measurements of laser; 2) High temperature extraction dilution and the forward scattering measurements of laser; 3) Particulates concentration measurement with alternating current (AC) coupling charge induction method. The market share of first two methods exceeds $90 \%$ [5].

- Direct extraction of high temperature gasification and the forward scattering measurements of laser technology

This technology takes PCME (PCME STACK 181 WS) and SICK (SICK FWE200) as main representative, the products of both companies are similar. The principle is shown in Fig. 1.2 [6]. The measured flue gas enters into measuring instrument from receive pipeline, then goes into measuring unit after heating by hot-air system and drop removal, purging fan is also equipped to keep the cooling and clean of laser probe[34]. After wet dust extraction, this method can effectively overcome the condensation and water drop influence monitored for in-situ particle.

FWE200 adopts the principle of forward scattering measurements of laser (Fig.1.3). Very low particle concentration can be detected because of the high sensitivity principle. A laser diode will emit a beam of modulated light with visual range (wavelength is about $650 \mathrm{~nm}$ ) towards the dust particle in flue gas. The scattered light by particle scattering is recorded 
by high sensitivity detector with about $15^{\circ}$ included angle with beam axle. The received signal will be transmitted to the measurement channel of microprocessor after amplification; this processor is the core component for measuring, controlling and evaluating electronic device. In flue, the measurement area is determined by overlap part of scattered beam and receives [7].

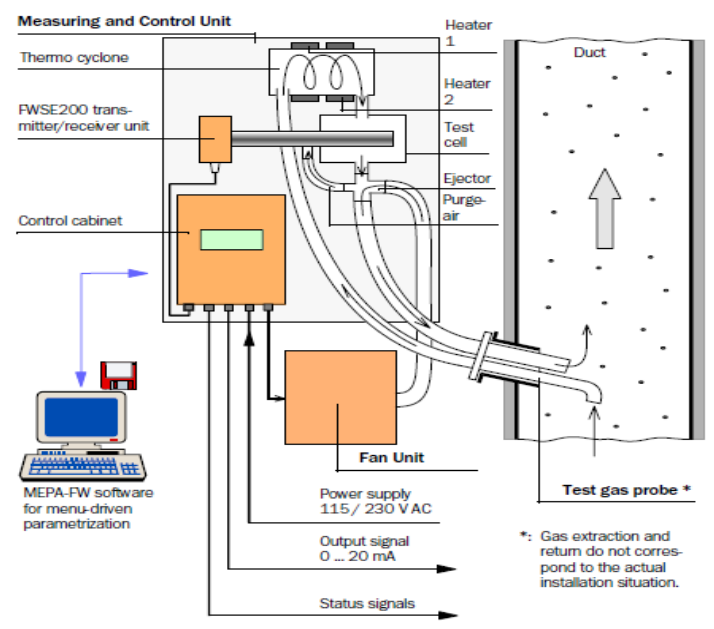

Fig.1.2 SICK FWE200 dust meter structure and schematic diagram

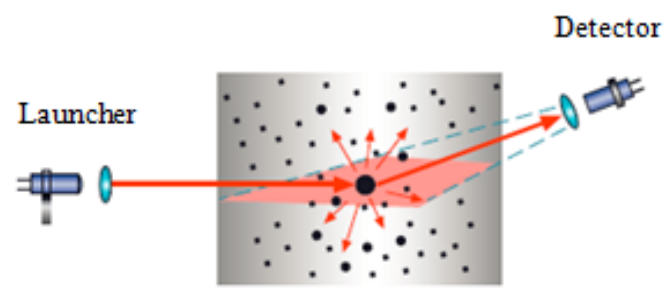

Fig.1.3 Laser forward scattering principle

- High temperature extraction dilution and laser forward scattering measuring technology

This technology mainly takes Durag and Fodisch as representative. The measurement principle is shown in Fig. 1.4. Sampling fan injects the air into jet-flow sampler at constant speed, jet-flow sampler will generate negative pressure to drainage the measured gas and diluents gas [12]. The measured gas mixes with diluent gas through high temperature heating sampling pipe, the diluent measured gas after mixing enters into measuring chamber via high-temperature ball valve, and conduct measurement with laser forward scattering measuring technology. Diluent gas pipe is equipped with flow rate and temperature sensor to accurately measure the diluent gas volume in unit time; the gas pipe is also equipped with flow rate and temperature sensor after dilution, the volume of measured gas in unit time after dilution can also be accurately measured; dilution ratio=gas mass of chamber in unit time after dilution mass of extracted sample gas in unit time $[8,9]$.

Use dilution method to measure particle concentration can reduce the dew point of flue gas; meanwhile the air can also be injected into sampling chamber through pump dilution to verify the zero point of the system. System has the function of automatic zero and full scale inspection, be able to timely find the problem of laser forward scattering measuring instrument pollution, laser attenuation.

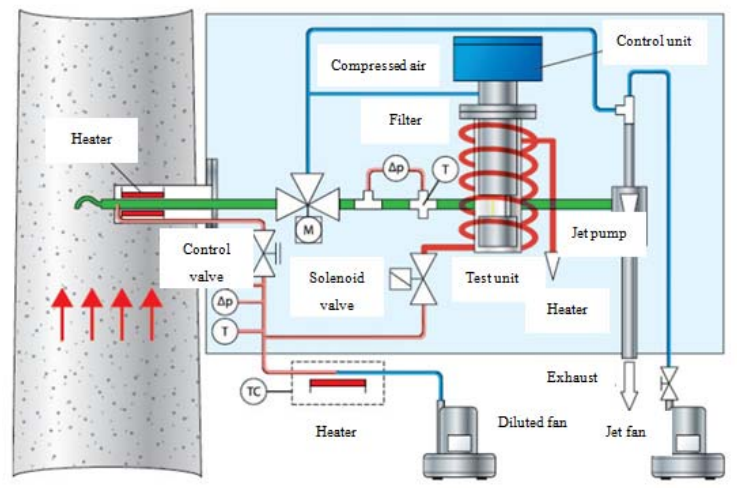

Fig.1.4 Durag company's D-R820 dust meter structure and schematic diagram

- AC coupling type charge induction particulates concentration measuring technology

This technology takes Finland (SINTROL) and Shanghai Lingcui as representative. The basic principle of technology: when the live dust particle passes near electrode, electrode surface will generate dynamic inductive charge (See Fig.1.5), the movable charge will generate alternating current (See Fig.1.6) [10], when a great deal of duct passes the inductive probe, it will cause signal superposition, the alternating signal volatility around mean value shall be analyzed by amplification and filtering, so as to reflect the size of particulates concentration (See Fig.1.7). Dust meter measures the particulates concentration by filtering out the DC signal by friction and contact, extract and analyze the AC signal on electrode due to charge induction [18].

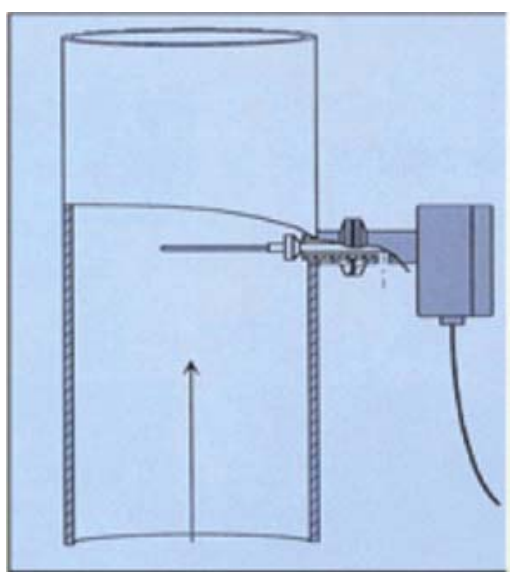

Fig.1.5 Schematic diagram of AC coupling dust meter 


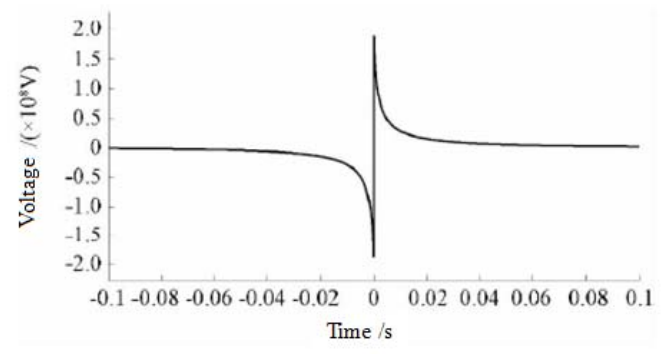

Fig.1.6 Alternating signal induced by unit charge:

The $x$-coordinate is time axis, the unit charge particle at $x=0$ is close to electrode surface, the $y$-coordinate is the voltage output by charge-amplifier. Thus, when each live dust particle moves along electrode radial direction, the charge-amplifier will output an alternating signal.

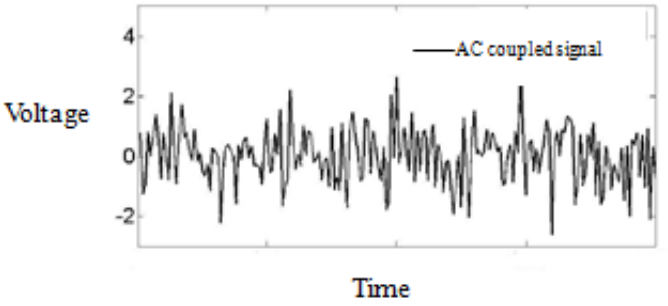

Fig. 1.7 AC coupling signal

Although AC coupling technology has better resistance to high humidity interference, its working condition in the flue gas environment with $80 \% \mathrm{RH}$ and higher humidity has a certain error. To improve the measurement accuracy of equipment, disposal for flue gas of dust sensor is needed [18].

\section{Other indirect measurement methods}

Other dust measurement methods also include $\beta$-ray and vibration scale method, etc, but these methods have higher requirements for measuring environment, and need constant temperature constant pressure, therefore these methods are mainly applied in monitoring of particle concentration in air, e.g. measuring base station, etc, and rarely used in monitoring of PM2.5 emission of power plant [6]. F-904-20 of Durag company is one of those $\beta$-ray method dust meter (Fig. 1.8). The type of dust meter is used to measure particulates concentration by measuring the $\beta$-ray in flue gas dust particle which is emitted from source of radiation. Adopt high temperature extraction dilution method to gather flue gas. See the follow fig. for dust meter structure schematic diagram. It has the function of automatic zero calibration and the accuracy is less than $5 \%$ of measuring scope [11].

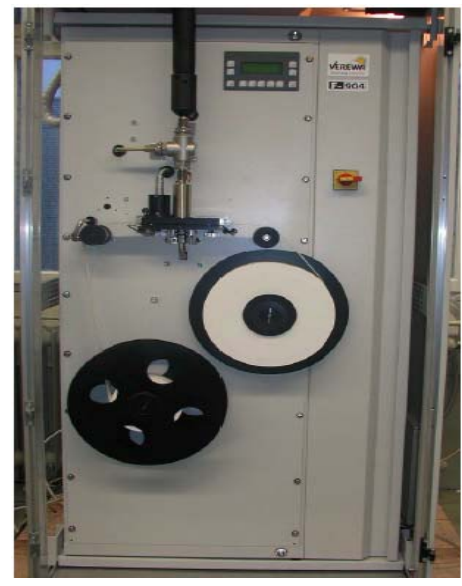

Fig. 1.8 Durag F-904-20 type of dust meter

\section{Technologies comparison}

For all the measurement methods above, they can theoretically realize effective measurement of dust content under the condition of high humidity and low concentration.

Because the indirect measurement method can realize continuously detecting online, so it is widely used in power plants, in which dust meters of SICK, Durag and PCME based on the basic principle of laser forward scattering measuring technology are more mature, and have passed the environmental certification of China National Environmental Monitoring Centre. The FWE200 type of dust meter of SICK shall be used for flue gas sampling, and continuous heating and heat preservation in the transmission process of flue gas, to prevent drops in flue gas and flue gas condensation in transmission process causing measurement errors. The D-R820 of Durag increases a set of diluent gas accessories on the basis, and the measurement environment is more stable. Contrast with laser forward scattering measuring technology, AC coupling charge induction type has more requirements for physical properties of dust, and especially after passing the WESP, the charge parameter of dust particle shall have changes, which effects on measurement results of the $\mathrm{AC}$ coupling charge induction type of dust meter are not easy to eliminate, so the measurement accuracy is difficult to guarantee. The $\beta$-ray and vibration scale method have higher requirements for measuring environment, and need constant temperature constant pressure, therefore, they are not be practically applied in power plant.

Compare with indirect measurement method, the direct measurement method can obtain the absolute mass concentration of flue particles, and the measured data is reliable, but the method has complex measuring process, and has higher requirement for operators. However, if adopting other methods for measuring, the weighing method is still the basic method of flue dust emission of fixed source. The accuracy of direct measurement method shall directly affect the accuracy of indirect measurement method including laser scattering method, therefore, the online measuring dust meter companies such as SICK and 
Durag develop their own high-accuracy weighing method measuring instrument as standard equipment of their own online measuring dust meter.

In conclusion, the accuracy of the direct measurement method represented by membrane weighing method is the highest, so this method is the first choice of dust meter for laboratory.

\section{MONITORING METHODS OF PM 2.5 ULTRAFINE PARTICLES}

The monitoring method is vital to the emission control of PM2.5 particles contained in the coal-fired flue gas. The stationary source monitoring consists of two steps: sampling and analysis.

\section{A. Sampling}

Up to now, there has been no special PM2.5 measuring method adopted in China while in the world, EPA (United States Environmental Protection Agency) ASTM (American Society for Testing and Materials), ECS (European Committee for Standardization) and ISO (International Organization for Standardization) have issued many standard sampling methods for PM 2.5 stationary-source particulates [1, 13-15, 36]. Generally, those methods can be classified into two kinds: direct sampling and dilution sampling.

\section{- Direct sampling method}

The direct sampling method for PM2.5 particulates in the exhaust gas from stationary pollution source is performed by such way that: insert the sampler into the flue directly, collect a given amount of flue gas at a constant speed at the temperature of flue gas, make the sample gas pass through the collecting medium and capture the PM2.5 particulates in the flue gas onto the collecting medium. The instrument used in this method is simple in structure and easy to operate. However, the direct sampling method is only applicable to the PM2.5 particulates that can be captured in the flue gas. The common direct sampling methods capture the PM2.5 particulates by classifying the particulates in the flue gas through inertia force or centrifugal force, and then collect PM2.5 with the collecting medium [16]. The main samplers include the inertia-type impactor, virtual impactor and cyclone. In order to ensure the accurate measurement of PM2.5 in the glue gas, all those samplers collect the samples at a constant flow almost equal to the flue gas flow in order to reduce the sampling deviation caused by the inertia motion of particulates.

1) Inertia-type impactor method (ISO 23210: 2009):

The inertia-type impactor method mainly uses the impact adhesion principles (Fig. 2.1) [27], and classifies the samplers as per their cutting size and in accordance with the impulse and inertia of particulates so that the sample of each grade can collect and cut the particulates in its cutting size range [13]. The dusty gas flows into the samplers and is injected out through the Grade 1 accelerating jet and blocked by the impact board, and then flows along a curved route in order to avoid the barrier; meanwhile, the particulates are separated from the gas flow under the effect of inertia force, and continue to move forward until the kinetic energy reduces to zero. At the same linear speed, the larger the size is, the higher the inertia force of particulate is and the higher the ability maintaining the former motion state is so it is easier to separate the particulates from the gas and capture the particulates onto the impact board due to the long forward moving distance [17]. Each grade of multi-grade impactor has a cutting size. The particulates with large cutting size are captured onto the impact board while the particulates with small cutting size flow to the next jet together with the gas, and are captured selectively until the rest particulates are captured by the large grade of filter membrane at the outlet $[18,19]$. The parameter representing the ability of particulates to move along with the gas flow is called as "Stokes number". If $50 \%$ of particulates of a certain size are captured by the collecting board, and the other $50 \%$ flow downstream together with the gas flow, this size is the cutting size (Dp50) of this inertia-type impactor, and the corresponding Stokes number is Stk50. The particulates with size smaller than Dp50 will mainly flow downstream along the gas flow while those with size bigger than Dp50 will mainly be captured by the collecting board. In this way, the particulates in the flue gas are classified [20-23]. Electrical Low Pressure Impactor (ELPI) and Dry Powder Inhalers (DPI) all use this technology.

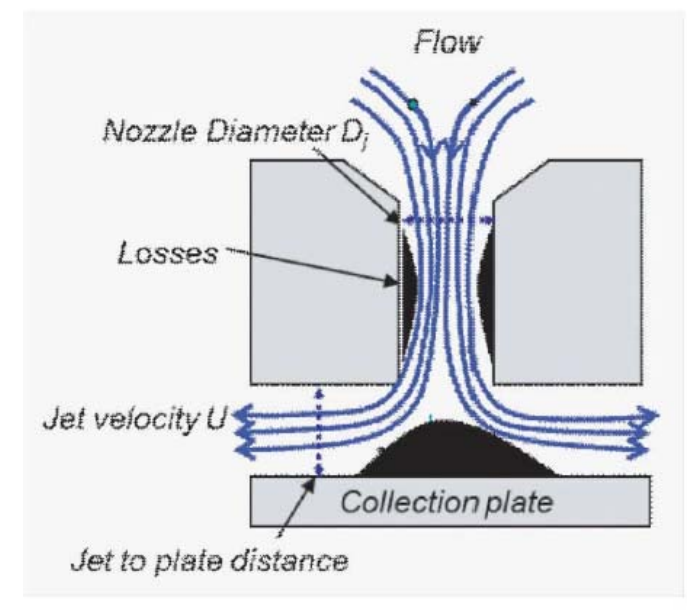

Fig. 2.1 Schematic diagram of inertia-type impactor

2) Virtual impactor method (ISO 13271: 2012):

As shown in Fig. 2.2, the sampling principle of virtual impactor method is similar with that of inertia-type impactor, namely sampling in grades through the different inertia forces of particulates with different sizes [36]. The difference between the two methods is that the glue gas is divided into major flow and minor flow in the inertia-type impactor, the particulates with small size and low Stokes number 
flow downward together with the major flow, and the particulates with large size and high Stokes number are separated, flow into the outlet along with the minor flow and are captured. For the graded cutting sampler of virtual impactor, it is necessary to control the multi-channel gas flow accurately, and the equipment configuration and control requirements are strict $[24,25]$.

The cutting effect of virtual impactor depends on its geometrical parameters and the ratio between the main and minor flows; the ratio between the main and minor flows determines the gradient of cutting efficiency curve; the geometrical parameters determine the loss of particulates, and the serious loss, if any, will affect the cutting efficiency curve [26]. The loss of virtual impactor mainly concentrates near the cutting point, and the actual loss mainly occurs at the top of outlet. The reasonably designed geometrical parameters of outlet top can reduce the loss below 5\% [31].

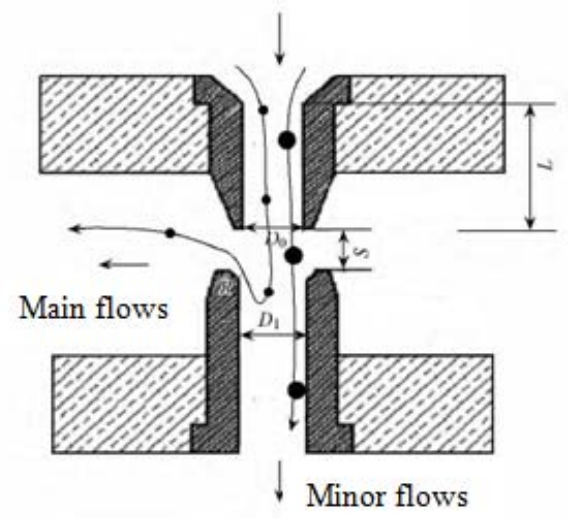

Fig. 2.2 Schematic diagram of virtual impactor

\section{3) Cyclone method (EPA 201A):}

The cyclone method is performed by such way that: force the gas flow to rotate and produce the centrifugal force so as to classify the particulates with different sizes. The large particulates are under a high centrifugal force and separated from the gas flow, and then deposit on the wall of cyclone while the small particulates are under a low centrifugal force, and flow out from the cyclone together with the gas flow. Fig. 2.3 shows the standard sampling method (EPA 201A) released by EPA [14]. Grade 1 PM10 cyclone is used to cut the particulates larger than $10 \mu \mathrm{m}$, and its flow route is different from the ordinary cyclone. The gas flow is blocked by the reversing cap on the top, and then flows into Grade 2 PM2.5 cyclone from the hopper. The PM2.5 cyclone has the same route as the ordinary cyclone, and is used to cut the particulates larger than $2.5 \mu \mathrm{m}$. Grade 1 cyclone and Grade 2 cyclone are connected through an elbow pipe. The particulates smaller than $2.5 \mu \mathrm{m}$ flow out from the outlet of Grade 2 cyclone together with the air flow and captured by the rear filter membrane [14]. After sampling, wash the hopper and cone surface of PM10 cyclone with acetone, then evaporate the acetone in the washing fluid, and the left are the particulates with
Da $>10 \mu \mathrm{m}$; in the same way, wash the hopper, cone and connecting pipe of PM2.5 cyclone with acetone, then evaporate the acetone, and the left are the particulates with $\mathrm{m}<\mathrm{Da} \leq 10 \mu \mathrm{m}$; The residuals in the outlet pipe of PM2.5 cyclone and on the rear filter membrane are the particulates with $\mathrm{Da} \leq 2.5 \mu \mathrm{m}$ [27]. During sampling, the PM10 and PM2.5 cyclones and the rear filter membrane are inserted in the flue to take samples; in this way, the PUM2.5 particulates that can be captured in the flue gas can be collected. The dust collecting surface of cyclone is much larger than the surface of connecting board of impactor so the overload can be avoided and the cyclone can be used to collect the high-concentration smoke with average value above $50 \mathrm{mg} \cdot \mathrm{m}-3$ for half an hour [28].
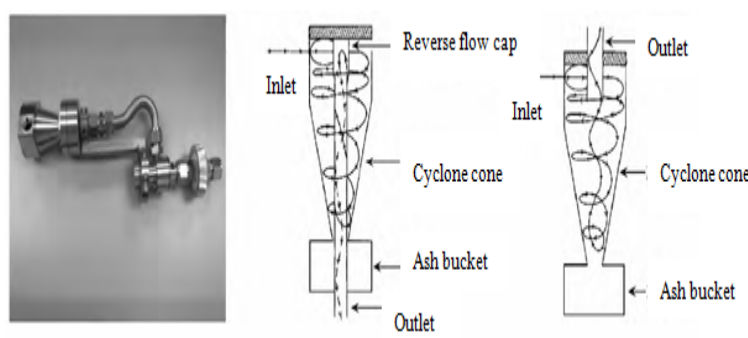

Fig. 2.3 Schematic diagram of two-grade cyclone

\section{- Dilution sampling method}

The principles of dilution sampling method are that: draw the high-temperature flue gas out from the flue through a sampling pipe, then mix the flue gas with the clean air without particulates to dilute and cool down the glue gas close to the ambient temperature, and finally measure the particulates by the common sampling method of atmospheric particulates. This method is based on the sampling method of atmospheric environment and simulates the mixing of high-temperature flue gas with the actual air after the flue gas flows out from the exhaust port so it is the method most close to the actual state of participates discharged from stationary pollution source in the atmospheric environment. After dilution, the collected PM2.5 particulates include the PM2.5 that can be captured and coagulated so its result is higher than the mass concentration of captured PM2.5 measured by the direct sampling method. Owing to the following advantages, the dilution sampling method is favored by the researchers [29, 30, 32]: 1) now the dilution sampling method has become the standard measuring method of emission factors of motor vehicles; 2) the particulate measuring condition in the dilution sampling method is similar to the smoke plume condition, and can be used to analyze the particulate source and evaluate its health effect; 3 ) the high temperature, high humidity and high pollution concentration of direct sampling method are avoided so the test method is diversified; 4) the conventional sampling method of atmospheric particulates can be used for sampling; 5) the on-line analysis technology of atmospheric particulates can be used to analyze the physical and chemical features of 
particulates in order to facilitate the establishment of source minutia feature; 6) the analysis process of the particulates that can be coagulated is simplified.

The main device used in the dilution sampling method is called as the dilution channel which is the core of this method. The main parameters of dilution channel include dilution ratio, dwell time and Renault number of mixing section. The principle of dilution channel is simple. The scholars from different countries have designed various dilution channels according to their needs, including positive-pressure dilution and negative-pressure dilution. In order to ensure the sufficient mixing of flue gas and diluting air, most of dilution channels use the turbulent mixing method [33, 35]. The dilution process is a dynamics process in which the volatile and semi-volatile substances interact with the particulates and the particulates interact with other particulates, including nucleation, coagulation and aggregation. There must be sufficient dwell time to ensure the stable distribution of particulates as per their sizes. After the research of many years, ISO proposed the dilution method standard (ISO25597: 2013) in 2013, and this standard uses the portable dilution channel designed by England, et al as the prototype. As shown in Fig. 2.4 [15], this method uses the two-grade PM10-PM2.5 cyclone to remove the particulates with aerodynamic diameter above $2.5 \mu \mathrm{m}$ in the flue gas, then dilutes and condensates the left glue gas, next uses a cyclone to remove the particulates with $\mathrm{Da}>2.5 \mu \mathrm{m}$ and uses the filter membrane to collect the PM2.5 particulates, and finally measure the weight [29]. This standard specifies the parameters of dilution channel: the dilution ratio shall not be below 20:1, the dwell time shall not be shorter than $10 \mathrm{~s}$, the temperature at the filter membrane shall not be above $42^{\circ} \mathrm{C}$ after diluting (Teflon filter membrane), and the relative humidity shall be below $70 \%$.

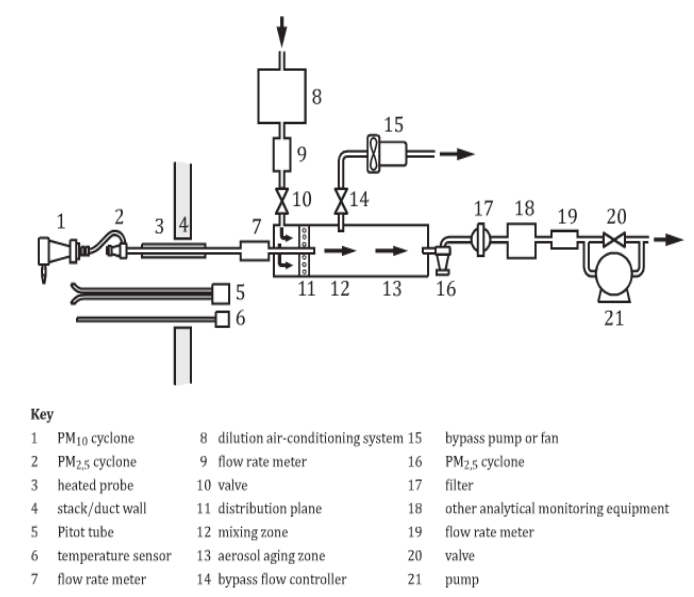

Fig. 2.4 In-stack PM 10 and PM 2.5 cyclones with dilution sampling train

\section{B. Analysis method}

(1) Weight method: This method is mainly applicable to DLP1 and DPI. Regardless of cyclone or impactor, the concentration of PM2.5 in the coal-fired flue gas is usually calculated by the weight method. By this method, the difference between the weight before and after sampling is measured and used in the calculation of the PM2.5 concentration in the coal-fired flue gas. This method has high requirements on the measuring accuracy.

(2) Electric charge method: This method is applicable to ELPI. The particulates with different sizes are collected in different grades, and the real-time size distribution of particulate concentration is reflected on the computer. ELPI can measure the distribution, number concentration, mass concentration and electric charge distribution of particulates with size within $0.03 \sim 10 \mu \mathrm{m}$ at real time and the measured results can be shown on the computer at real time.

(3) Other methods: Other analysis methods, such as $\beta$-ray absorbing method and micro-vibration balance method, are mainly used to monitor the particulate concentration in the air.

\section{Method comparison}

The technical difficulty of graded monitoring of PM2.5 particulates concentration is the graded sampling. The inertia-type impactor is only applicable to the glue gas whose average concentration in half a hour is not above $40 \mathrm{mg} \cdot \mathrm{m}-3$, and not suitable for the glue gas with high particulate concentration or the glue gas with saturated steam, such as the glue gas at the wet FGD outlet because the condensation of steam will change the aerodynamic behaviors of particulates.

The diameter of existing virtual impactor is larger than the stationary-source flue gas sampling port $(80 \mathrm{~mm})$ used in China so that it cannot be inserted directly into the flue, and an extended gooseneck tube shall be used outside the flue for sampling, which will increase the loss of particulates. Compared with the conventional impactor, the virtual impactor has another two flows, making the operation more complex. Up to now, none report about the commercialization of virtual impactor has been found.

The maximum limit of graded cyclone sampling method is that it is necessary to wash the whole cyclone with acetone, evaporate the acetone in the washing fluid and measure the weight after sampling. The whole process is complex and the grades are fewer, normally just $10 \mu \mathrm{m}$ and $2.5 \mu \mathrm{m}$.

The dilution sample method takes the samples outside the flue so a pipe is needed to draw the flue gas out, which will result in the loss of particulates. The main loss mechanisms include gravity setting, inertia collision, diffusion loss, static loss and thermo-phoretic force loss. 
Moreover, all the graded sampling methods distinguish the diameters of particulates by aerodynamics, and are not applicable to the flue gas with saturated water so the water shall be removed by pretreatment before staging. There are two kinds of pretreatment devices: diffusion dryer and dilution channel.

The graded particulates are mainly measured by weight method and electric charge method. The weight method directly reflects the result and has high requirements on the accuracy so the negative value often occurs during actual measurement. The electric charge method calculates the result by the empirical formula and has the following advantages: the results can be obtained online, and after the parameters of test environment are calibrated, the related results are true and reliable.

\section{CONCLUSIONS AND RECOMMENDATIONS}

For the measurement of low concentration particulates in high humidity flue gas, the simple and convenient indirect measurement methods, such as the forward scattering measurements of laser technology and $\mathrm{AC}$ coupling type charge induction particulates concentration measuring technology, are suggested with the advantage of $24 / 7$ continuously measuring ability. For a more accurate quantitative measurement, it is highly recommended to apply the membrane weighing method, which is also used as the calibration of the indirect measurement methods mentioned above.

For the measurement of PM2.5 ultrafine particles, the inertia-type impactor methods such as ELPI or DPI are extensively used, especially in the sampling process of stationary pulverized coal-fired power plants. It is easy to operate compared to virtual impactor method and also more accurate than cyclone method. As two of the most popular measuring devices, more detailed comparisons between ELPI and DPI are explained as below.

ELPI has the following advantages: (1) the detailed grade information of particulates can be obtained, in which totally 13 grades are included; 2) when the parameters of tested glue gas are stable, the measurement result obtained by the electric charge method are accurate and reliable, and it is easy to read the result; 3) when necessary, ELPI can measure the weight directly by the weighing method. Owing to those advantages, ELPI is used by the institutes and universities as the basic measuring method for the research of dust particulates.

Compared with ELPI, DPI weakens the grading ability of particulates, and only has 3 grades, and cannot make measurement by the electric charge method. However, DIP has the following advantages that ELPI does not have: 1) the weighing workload is simplified and the weighing error is reduced; 2) DPI can directly measure the high-temperature gas, has no high requirements on the measured flue gas, and has a strong applicability. Owing to those advantages, DPI is used as the measuring method in the engineering projects.

\section{REFERENCES}

[1] GB 16157-1996, Determination of Particulates and Sampling Methods of Gaseous Pollutants from Exhaust Gas of Stationary Source [S]. 1996.

[2] GB/T 13931-2002, Methods of Performance Tests for Electrostatic Precipitators [S]. 2002.

[3] Wang Zhichao, Wu Zhansong, Yang Yingxia, and Li Jiandong, Experiment Research of Mass Concentration Test Method of Dust [J], Tsinghua Science and Technology, 2013 (3): 366-370

[4] Yang Ding, Chen Weixiang, and Zheng Fang, Studies on low concentration of dust emission test in coal - fired flue gas [J], Electric Power Environmental Protection, 2015, 31 (5): 28-31.

[5] Wu Chaofeng, Application of Wet-type Dust Removing and Monitoring Technology with Ultra-low Emission in Thermal Power Plant [J], Science and Technology Innovation Herald, 2015 (27): 75-79.

[6] He Zhenjiang, Yang Guanling, Wu Kaihua, Huang Zuohua, Han Peng, and Yu Yongchang, RESEARCH ON MEASURING CONCENTRATION PROBLEMS IN A NEWLASER SUPERVISING SYSTEMOF FUNNEL ASH UANTITY $[\mathrm{J}]$, Journal of South China Normal University(Natural Science Edition), 2000 (2): 1-5.

[7] Liu Xiaohu,Study on the Calibration Technology of Measuring Dust Concentration by Light Transmission Method,Mechanical and Electronics,2014(10):52-55

[8] LI Kun, ZHONG Lei, ZHANG Hong-quan, Review of smoke concentration measurement method [J],Transducer and Micro system Technologies, 2013, 32(2):1-5

[9] Lei Zhiwei, STUDY OF MEASUEMENT SYSTEM FOR DUST CONCENTRATION BASED ON LASER EXTINCTION [D], Nanjing, Southeast University, 2015.

[10] Lin Chundai, Research of Online Monitoring System of Flue Gas with High Humidity and Its Application in Wet Flue Gas Desulfurization $[R]$, Shanghai, Shanghai Lingcui Environmental Protection Technology Co. Ltd., 2013.4.

[11] Zhang Xiaochun, Zhao Yanan, and Jia Xiaofang, Comparison Analysis of PM2.5 Mass Concentration in Aerosol by Membrane Sampling and $\beta$-ray Method [J], Journal of Atmospheric and Environmental Optics, 2015, 10(2): 149-157.

[12] Ye Chao, Meng Rui, and Ge Baozhen, Overview of Particulate Measuring Method Based on Light Scattering [J], Laser and Infrared, 2015 (4): 343-348.

[13] ISO 23210: $2009, \quad$ Stationary source emissions-Determination of PM10 /PM2. 5 mass concentration in flue gas --Measurement at low concentrations by use of impactors [S] . 2009.

[14] USEPA Method 201a and 202, Methods for measurement of filterable PM10 and PM2. 5 and measurement of condensable particulate matter emissions from stationary sources $[\mathrm{S}]$, 2010.

[15] ISO 25597: 2013, Stationary source emissions-test method for determining PM2. 5 and PM10 mass in stack gases using cyclone samplers and sample dilution [S] . 2013.

[16] Xie Ruijia, Research of Development and Measured Date Accuracy of PM2.5 Monitoring Technology [J], Science and Techology of West China, 2015 (9): 106-109.

[17] Huang Binxiang, Shu Jiahua, and Sun Lingling, Content Measurement of Fine Particulates (PM2.5) in Gas with Low Particulates concentration [R], Shanghai, Shanghai Lingqiao Environment Protection Equipment Works Co., Ltd., 2013.11: 25-28.

[18] Chen Jiange, Wang Jiewu, and Fu Xiang, Research of Dust Concentration Test Technology by AC Coupling Charge Inductive Method [J], Monitoring and Evaluation: 131-147.

[19] Wang Aimin, Pollutant Emission Measurement and Instrument Selection Analysis of Power Plant [J], Inner Mongolia Petrochemical Industry, 2013 (9); 77-78.

[20] Yuan Yongtao, Wei Yuzhen, Zhang Binwei, Qi Liqiang, Research of Application of Multi-grade Impactor in Sampling of Flue Ash of Power Plant [J], Thermal Plant, 2010 (5): 77-81. 
[21] Liu Xiaohu, Research of Particulates concentration Measuring Technology [D], Xi'an, Xi'an Technology University, 2013.4.

[22] Liu Tao, Zhang Jian, and Guo Shengjun, Research and Development of Dust Concentration On-line Monitoring and Dust Removing Device [J], Coal Mine Machinery, 2015 (10): 193-196.

[23] $\mathrm{Hu}$ Yueqi, Wang Chen, Wu Xiaodong, and Li Meng, Status Analysis and Consideration of Monitoring Technology of Particulates from Stationary Source [J], Environmental Science and Technology, 2016,29 (1): 69-74.

[24] Wada M, Tsukada M, Kondo A, et al. Separation characteristics of a multi-stage VIS impactor for PM10/PM2. 5 mass concentration measurement in a stack of a stationary source $[\mathrm{J}]$. Journal of the Society of Powder Technology, Japan, 2009, 46( 6) : 467-475.

[25] Marple V A, Willeke K. Impactor design [J]. Atmos. Environ, 1976, 10:891-896.

[26] Jiang Jingkun, Deng Jianguo, Duan Lei, Zhang Qiang, Li Zhen, Chen Xiaotong, Li Xinghua, and Hao Jiming, Development of a Two Stage Virtual Impactor for Stationary Source PM10 and PM2. 5 Sampling [J], Acta Scientiae Circumstantiae, 2014,35 (10): 3639-3643.

[27] Wang Zheng, Xue Jianming, Xu Yueyang, Wang Hongliang, Wang Xiaoming, Guan Yiming, and Liu Jun, Research on Testing Methods for PM 2. 5 Emission in Coal-fired Power Plants [J], Journal of Environmental Engineering Technology, 2013, 3(2):

[28] Jiang Jingkun, Deng Jianguo, Li Zhen, Li Xinghua, Duan Lei, and Hao Jiming, Sampling Methods for PM2. 5 from Stationary Sources: a Review [J], Acta Scientiae Circumstantiae, 2014 (5): 2018-2024.

[29] Hu Yuanzhi, Hou Banglei, Ma Bo, and Xujin, Research and Application of Sampling Test Methods of PM10/PM2.5 Particulates in Exhaust Gas of Stationary Source [C], Academic Annual Conference Proceedings of Chinese Society for Environmental Sciences, 2014: 4753-4757.

[30] Kong Shaofei, Bai Zhipeng, Lu Bing, Han Bin, and Guo Guanghuan, Research Development of Sampling Methods of Particulates from Stationary Source [J], Environmental Science and Technology, 2011,34 (12): 88-94.

[31] Marple V A, Chien C M. Virtual impactors: A theoretical study [J]. Environmental Science and Technology, 1980, 14 ( 8 ) : 976-984.

[32] 133-137.

[33] Li Xinghua, Duan Lei, Hao Jiming, Yi Honghong, and Guo Xingming, Design and application of a dilution stack sampling system for measuring particulate matter [J], Acta Scientiae Circumstantiae, 2008 (3): 458-463.

[34] Liu Shuai, Deng Shuang, Huang Jiayu, Tian Gang, Wang Linlin, Chen Xiaopeng, and Zhang Fan, Primary PM2.5 Monitoring and Control Technology of Coal-fired Boiler [J], Energy and Environment, 2015 (1): 25-30.

[35] Chen Huan, Chang Qianyun, and Xu Juanjuan, Research and Application of Test Method of Ultra-low-concentration Particulates in Coal-fired Flue Gas [C], Academic Annual Conference Proceedings of Chinese Society for Environmental Sciences, Beijing, 2015: 3310-3314.

[36] Tian Dongmei, Jiang Zhong'an, Yao Jian, and Niu Wei, Comparison between Chinese and Foreign Dust Monitoring Technologies [J], Metal Mine, 2008 (7): 116-119.

[37] ISO 13271: $2012, \quad$ Stationary source emissions-Determination of PM10 /PM2. 5 mass concentration in flue gas -Measurement at higher concentrations by use of virtual impactors [S] . 2012. 